\title{
IPv6 Functional Test on Mainstream Smartphones
}

\author{
Gang Qin, Can Chen, Baoping Yan \\ Computer Network Information Center, Chinese Academy of Sciences \\ 4, 4th South Street, Zhongguancun, Haidian District \\ Beijing, China \\ gqin@cnic.cn
}

\begin{abstract}
Nowadays, Internet has become an indispensable part of our life. Smartphone has become an important device to access Internet. Hundreds of millions of smartphones need a large number of IP addresses. IPv6 instead of IPv4 can meet the IP address requirement. IPv6 also has many advantages compared to IPv4. In China, IPv6 research and construction has been carried out for many years and have yielded a great deal of fruits. While application of IPv6 is far from sufficient. Smartphone may be the killer application field of IPv6. In this paper, we test IPv6 functions on mainstream smartphones which run different Operating Systems, including Android, iOS, MeeGo, Symbian, BlackBerry. Then we give the test result and look forward the future work.
\end{abstract}

Keywords-IPv6; Smartphone; Android; iOS

\section{INTRODUCTION}

Nowadays, Internet has become an indispensable part of our life. Many kinds of devices are used to surf online every day. People are not satisfied to use PC indoors or carry a heavy notebook outdoors. With the development of mobile communications technology and integrated circuit technology, mobile phones are becoming more and more powerful. Mobile phones are not only capable of calling, sending SMS, but also able to access Internet. Smartphones have emerged and popularized very quickly.

A smartphone is a mobile phone built on a mobile operating system, with more advanced computing capability and connectivity than a feature phone[1]. Many modern smartphones include high-resolution touchscreens and web browsers that display standard web pages as well as mobile-optimized sites. High-speed data access is provided by Wi-Fi and mobile broadband. In recent years, the rapid development of mobile app markets and mobile commerce has been drivers of smartphone adoption [2].

Meanwhile, the need of hundreds of millions of smartphones accessing Internet will require a large quantity of IP addresses. The address space of the existing Internet Protocol - IPv4 is very limited, which has 232 addresses. And IPv4 addresses have been exhausted [3]. IPv6 has been brought out since 1990s. In IPv6, the address space is 2128 which is almost unlimited avoiding shortage of address. IPv6 also has many advantages compared with IPv4, such as improving process efficiency by simplifying and fixing the basic packet header, adopting hierarchical address format to make route aggregation much easier, plug and play auto configuration, enhanced security by using IPSec, supporting mobility for Mobile IP, etc.

In China, IP address shortage is more serious than other countries. So the research and construction of IPv6 have been carried out since 2003. The renowned project CNGI (China Next Generation Internet) is one of the fruits. Although the backbone and Custom Premises Networks (CPN) have been constructed to support IPv6, the application of IPv6 is still not up to expectations. Shortage of users and applications has become the most important obstacle preventing the development of IPv6.

Smartphones have the ability to access network and can run many interesting applications based on network connection. So we think smartphones may be the killer application field of IPv6.

This paper is organized as follows. Section 1 gives introduction of this paper. Section 2 introduces test schemes. Section 3 illustrates the tests procedure. Section 4 gives the conclusion. Section 5 looks forward the future work.

\section{TEST SCHEMES}

\section{A. Test devices}

In this paper, we select Lenovo A750 which running Android and IPhone4 which running iOS. Considering Nokia mobile phones have a large number of users, we select N9 which running MeeGo and E7 which running Symbian Belle. In the scope of business, BlackBerry mobile phones have a certain volume of users. So we also select BlackBerry 9860 as a test device. Table I shows the test devices.

TABLE I. TEST DEVICES LIST

\begin{tabular}{|l|l|l|}
\hline No. & Device Name & OS Version \\
\hline 1 & Lenovo A750 & Android 2.3 \\
\hline 2 & IPhone4 & iOS 4.0 \\
\hline 3 & Nokia N9 & MeeGo 1.2 \\
\hline 4 & Nokia E7 & Symbian belle \\
\hline 5 & BlackBerry 9860 & BlackBerry 7.1 \\
\hline
\end{tabular}

\section{B. Test Environment}

Different from PC and Notebook, smartphones cannot support wired network connection. They can only access mobile communication network or wireless network. 
Because lack of IPv6 support from mobile communication carriers, we have to construct a wireless network environment supporting IPv6. Considering insufficient support for IPv6 of some OS and some functions relying on IPv4, such as DNS resolving, we construct a wireless network environment both supporting IPv4 and IPv6.

According to our investigation, most wireless routers on the market only support IPv4 at present. Only a little of products which support IPv6 transparent transmission can support IPv6. Transparent transmission means that whatever services are being transmitted, the device will transmit service from source to destination ensuring the quality of transmission while don't process the service transmitted. Wireless routers supporting transparent transmission are capable of transparently transmitting IPv6 protocol packets receiving from uplink, especially IPv6 prefix advertisement messages, to network terminal devices connected to them. With this technology, these devices can access IPv6. In this paper, we use WHR-G300N v2 wireless router manufactured by Buffalo Technology Inc. to construct WIFI network environment.

Figure 1 shows the test environment. The details are as follows:

- A third layer switch uplinks to CNGI network, which supporting both IPv4 and IPv6;

- The switch also acts as the IPv6 gateway of the test environment which advertising IPv6 prefix advertisement messages;

- A wireless router uplinks to the switch;

- Test devices turn on WIFI and connect to the wireless router;

- IP video camera which supporting IPv6 is used in application test.

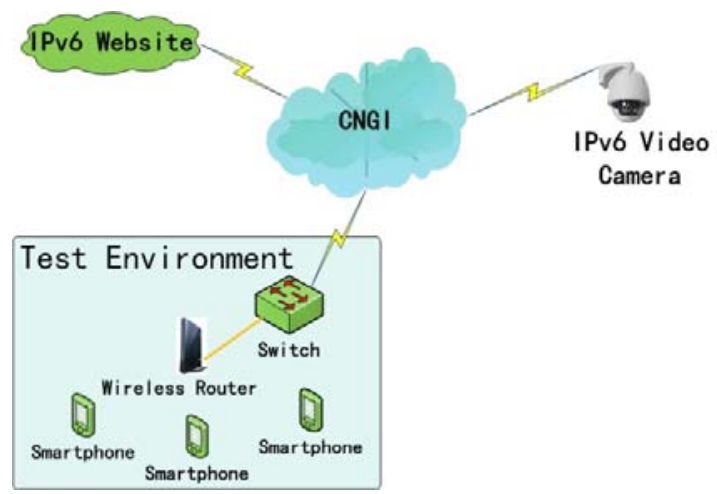

Figure 1. Test Environment

\section{Test Cases}

Two types of test cases are adopted in our test. One is IPv6 basic function test. The other is application test.

- IPv6 Basic Function Test

In IPv6 basic function test, we will test whether the device can get IPv6 address and can visit the famous IPv6 website http://www.kame.net by IPv6 which can be verified by a dancing turtle.

- IPv6 Application Test
In IPv6 application test, we will use an IP video camera supporting IPv6 installed in the Qinghai Lake Reserve to test whether the test device can connect to the camera successfully.

\section{TEST PROCEDURES}

\section{A. Lenovo A750}

Turn on WIFI on A750. Connect to the wireless router to get IP addresses automatically. Install the app "IPv6Config" on thisphone. Run this app. IP address information of the phone including IPv4 address and IPv6 address will be shown. As shown in Figure2, we can see the phone has got IPv6 address successfully. During the test, we find that only IPv4 DNS server instead of IPv6 DNS server can be set in the phone. So DNS resolutions have to be processed with IPv4.

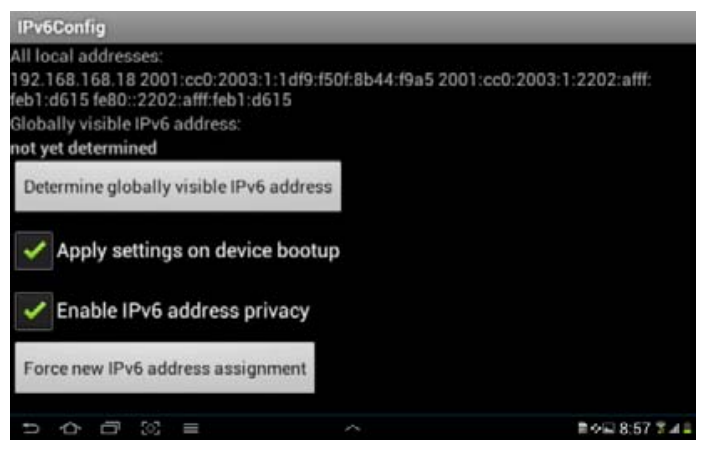

Figure 2. IPv6 Address Information

Visitthe homepage of Kame website through browser of the phone. It shows a dancing turtle which verifies A750 is accessing the website by IPv6.

Install the app "IP Cam Viewer" in the phone. Add the IPv6 address of the IP video camera in the app. As shown in Figure 3 , the phone can connect to the video camera and display the real-time video image successfully.

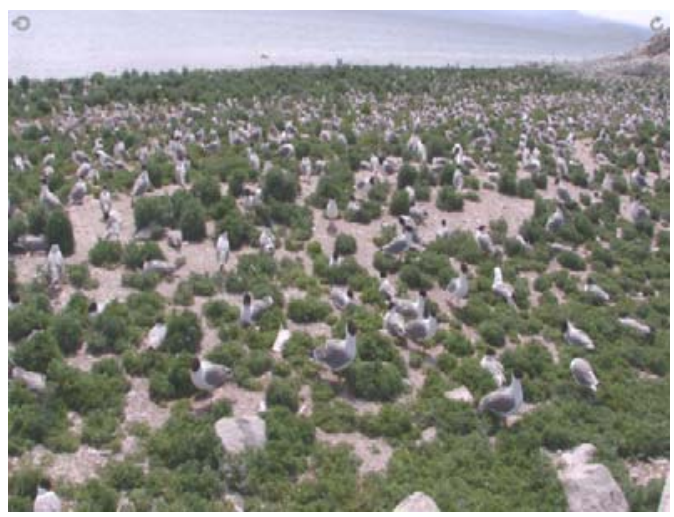

Figure 3. Real-time Video

\section{B. IPhone4}

Turn on WIFI in IPhone4. Connect to the wireless router to get IP addresses automatically. During the test, we couldn't find an app that can show IPv6 address information 
of IPhone4. However, as shown in Figure 4, we can set IPv6 DNS server in IPhone4 network configuration interface. So in IPhone4, we could use IPv6 DNS server to resolve domain names.

Visit the homepage of Kame website through browser. We can see a dancing turtle. It verifies that IPhone has got IPv6 address and can visit website through IPv6.

Install the app "uVivotek" on IPhone4. Add the IPv6 address of the IP video camera in this app. IPhone4 could connect to this camera and display the real-time video image successfully.

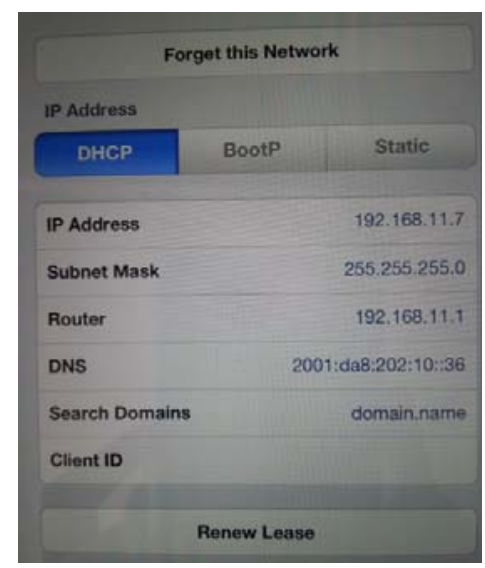

Figure 4. Network Configuration of IPhone4

\section{Nokia N9}

N9 can support IPv6 according to the test. While N9 should turn on the "Developer Mode" and install related protocols and tools. The detailed steps are as follows: (1) Enter "Configuration”-“Security”-“Developer Mode”; (2) Turn on "Developer Mode"; (3) Install "IPv6". After these steps, turn on WIFI on N9. Connect to the wireless router to get IP addresses. We could find the IPv6 address of N9 by the command line tool in Developer Mode, as shown in Figure 5.

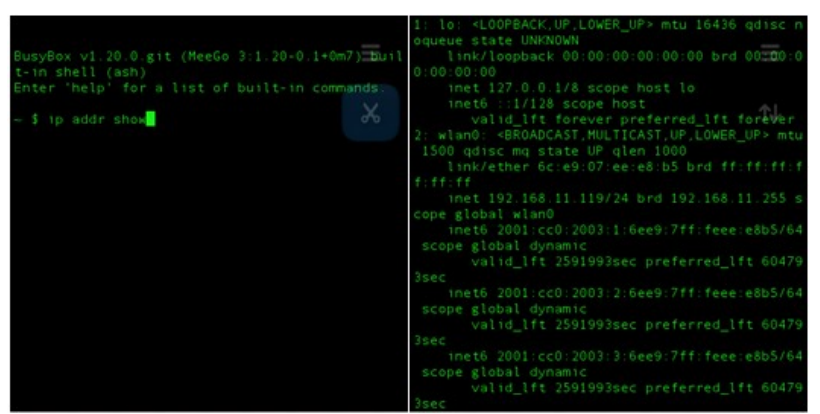

Figure 5. IPv6 Address of N9

Visit the homepage of KAME website. The dancing turtle on the webpage verifies that $\mathrm{N} 9$ is connecting to the homepage through IPv6.

We can connect to the video camera through IPv6 in web browser. Because none app of MeeGO which supporting the video stream format of IP video camera is found, we couldn't show the real-time video image on N9.

\section{Nokia E7}

The official document of Nokia said that Symbian is supporting IPv6 [4]. During the test, we find IPv6 DNS configuration in E7 network configuration interface. But we couldn't find the way to show whether E7 has got IPv6 address. When we connect to the KAME website, it shows a still turtle which verifies E7 is connecting to the website by IPv4 instead of IPv6.

\section{E. BlackBerry 9860}

The test shows that 9860 running BlackBerry 7 doesn't support IPv6. Neither can it get IPv6 address nor can visit IPv6 website.

\section{CONCLUSIONS}

Table II shows the test results.

TABLE II. TEST RESULTS

\begin{tabular}{|l|l|l|l|}
\hline No. & \multicolumn{1}{|c|}{ OS } & Device Name & IPv6 Capabilities \\
\hline 1 & Android & Lenovo A750 & Supporting \\
\hline 2 & iOS & IPhone4 & Supporting \\
\hline 3 & MeeGo & Nokia N9 & Supporting \\
\hline 4 & Symbian & Nokia E7 & Partial Supporting \\
\hline 5 & BlackBerry & 9860 & Not Supporting \\
\hline
\end{tabular}

We should give some explanations to Table II.

Android supports IPv6 since Android 2.2. While manufactures of devices maybe drop IPv6 module in official ROMs, IPv6 capability depends on the specific device. Even for the IPv6 supported device, it should be rooted firstly. Android rooting is the process of allowing users of smartphones to attain privileged control (known as "root access") within Android's subsystem. After rooting, it still needs to install apps like IPv6Config to turn on IPv6 functions.

iOS supports IPv6 since iOS 4.0. Devices running iOS could access Internet through IPv6 successfully.

BlackBerry for smartphone doesn't support IPv6 at present. While according to related resources [5], PlayBook OS running on BlackBerry Tablet, named PlayBook, supports IPv6.

\section{FUTURE WORK}

In this paper, none of Windows Phone has been tested. According to related resources [6], Windows Phone can support IPv6 since Windows Phone 7 Mango OS. And Windows Phone 8 will fully support IPv6. Considering the good interoperability with Windows OS running on PC and Notebook, we will test Windows Phone in future.

IPv6 instead of IPv4 is adopted in 4G mobile communications [7]. 4G networks have been constructed in many countries and regions. With the popularization of $4 \mathrm{G}$ network, more and more smartphones will access Internet through IPv6. We will test IPv6 service conditions of smartphones under $4 \mathrm{G}$ network.

\section{ACKNOWLEDGMENT}

This research is supported by the project of Scientific Research Infrastructure Construction and Typical 
Demonstrations based on CNGI, which is sponsored by National Development and Reform Commission (NDRC) of China, and National “973” Project (no. 2009CB320502).

\section{REFERENCES}

[1] Andrew Nusca (20 August 2009). "Smartphone vs. feature phone arms race heats up; which did you buy?". ZDNet. Retrieved 2011-12-15.

[2] http://en.wikipedia.org/wiki/Smartphone

[3] Smith,Lucie;Lipner,Ian. "Free Pool of IPv4 Address Space Depleted". Number Resource Organization. Retrieved 3 February 2011.
[4] http://www.developer.nokia.com/info/sw.nokia.com/id/a2586337-bd1 a-4d96-92bd-5959248b73e8/Nokia_Views_on_IPv6_Transition_v2_ 4_en.pdf.html

[5] http://www.progweb.com/en/2011/11/BlackBerry-playbook/

[6] http://wmpoweruser.com/windows-phone-only-getting-ipv6-supportwith-apollo/

[7] Gazis, V.; Housos, N.; Alonistioti, A.; Merakos, L. Generic system architecture for $4 \mathrm{G}$ mobile communications. The 57th IEEE Semiannual Volume 3, 22-25 April 2003 Page(s):1512 - 1516 vol.3.3. 